\title{
Hallucinating Facial Image Based on Adaptive Neighbourhood Selection
}

\author{
Fang $\mathrm{Liu}^{1}$, Yu Deng ${ }^{2}$ \\ ${ }^{1}$ China ship development and design center, Wuhan 430064, People’s Republic of China \\ ${ }^{2}$ Department of Electronic Engineering, Tsinghua University, Beijing 100084, People’s Republic of China
}

\begin{abstract}
In most manifold learning based face hallucination algorithms, the nearest neighbourhood metric is often adopted to describe the face subspace, which could not accurately capture the local geometrical structures of the samples. In this paper, a novel face superresolution approach based on adaptive neighbourhood selection is presented, which can adaptively select the nearest neighbours for each sample point. The corresponding neighbours of sample points well reflect the local geometrical structure of the face manifold, so that the linear subspace determined by the optimal linear fitting can approximate the local geometry well. Experimental results show that our method is more effective than other manifold learning based strategies for super-resolving face images.
\end{abstract}

Keywords- face hallucination; face resolution; adaptive manifold learning.

\section{INTRODUCTION}

High resolution (HR) face images are the key for many real applications such as video surveillance, automatic identity authentication, etc. However, in many cases, the facial images captured by the digital cameras are at a low resolution (LR) due to the limitations of imaging equipments or imaging environments. The LR facial images will degrade the performance of face recognition system seriously [1], generating the HR face image from its LR inputs has become one of the most important research regions for face recognition and analysis.

In recent years, manifold learning has drawn considerable attentions in the area of image superresolution. A great number of face super-resolution, or face hallucination, approaches based on manifold learning have been proposed. For instances, Chan et al. [2] presented a face hallucination strategy through neighbour embedding. In [3], Wang et al. introduced a global face hallucination scheme based on eigentransformation. Huang et al. [4] presented how to hallucinate the HR face images by using canonical correlation analysis. In order to hallucinate the facial images of higher quality at a lower computational cost, Ma et al. [5] proposed a sparse representation and position prior based face superresolution method. Recently, Jiang et al. [6] introduced a face hallucination algorithm based on near feature line (NFL) embedding, which improved the generalized ability of the training sets.

The above mentioned face hallucination strategies based on neighbourhood embedding often rely on the conclusion that the similar local geometric structures relationship between the HR and LR facial manifolds can be hold with the optimal selected neighbourhood, and the local geometry of the face manifold can be described as linear. That is, the local manifold geometry can be linearly approximated within the neighbourhood of each face patch (feature). According to this assumption, the $k$-nearestneighbourhood $(k$-NN) or $\varepsilon$-neighbourhood algorithm is commonly exploited to select the neighbours for a given LR patch in existing approaches. However, the neighbourhood with fixed size cannot reflect the variation in the curvature of the manifold efficiently nor its interplay with the data sampling density [7]. Thus, these neighbourhoods utilized in aforementioned facial hallucination approaches can not reflect the local geometry of training samples well.

In this paper, we first briefly introduce the adaptive neighbourhood selection (ANS) method, and then propose a face hallucination algorithm based on ANS. It can select the optimal neighbours to match the local geometry of the training examples adaptively. In other words, our algorithm can better capture the local geometric relationship of facial manifold than other approaches. As a result, the linear subspace determined by the optimal linear fitting can approximate the local geometry with high accuracy. Extensive experiments have confirmed the effectiveness and good performance of our algorithm.

\section{A BRIEF REVIEW OF ANS}

In many manifold learning algorithms, one of the key issues needed to be addressed is what can be determined as local. This challenging problem was briefly discussed by both S. Roweis et al. [7] and Zhang et al. [8]. Unfortunately, no practical solutions have been given in their studies.

To solve this problem, an adaptive neighbourhood selection criterion called neighbourhood contraction has been introduced by Zhang et al., which can adaptively select the optimal neighbour set for each sample point [9]. In our study, the improved adaptive neighbourhood selection procedure is summarized as follows: 


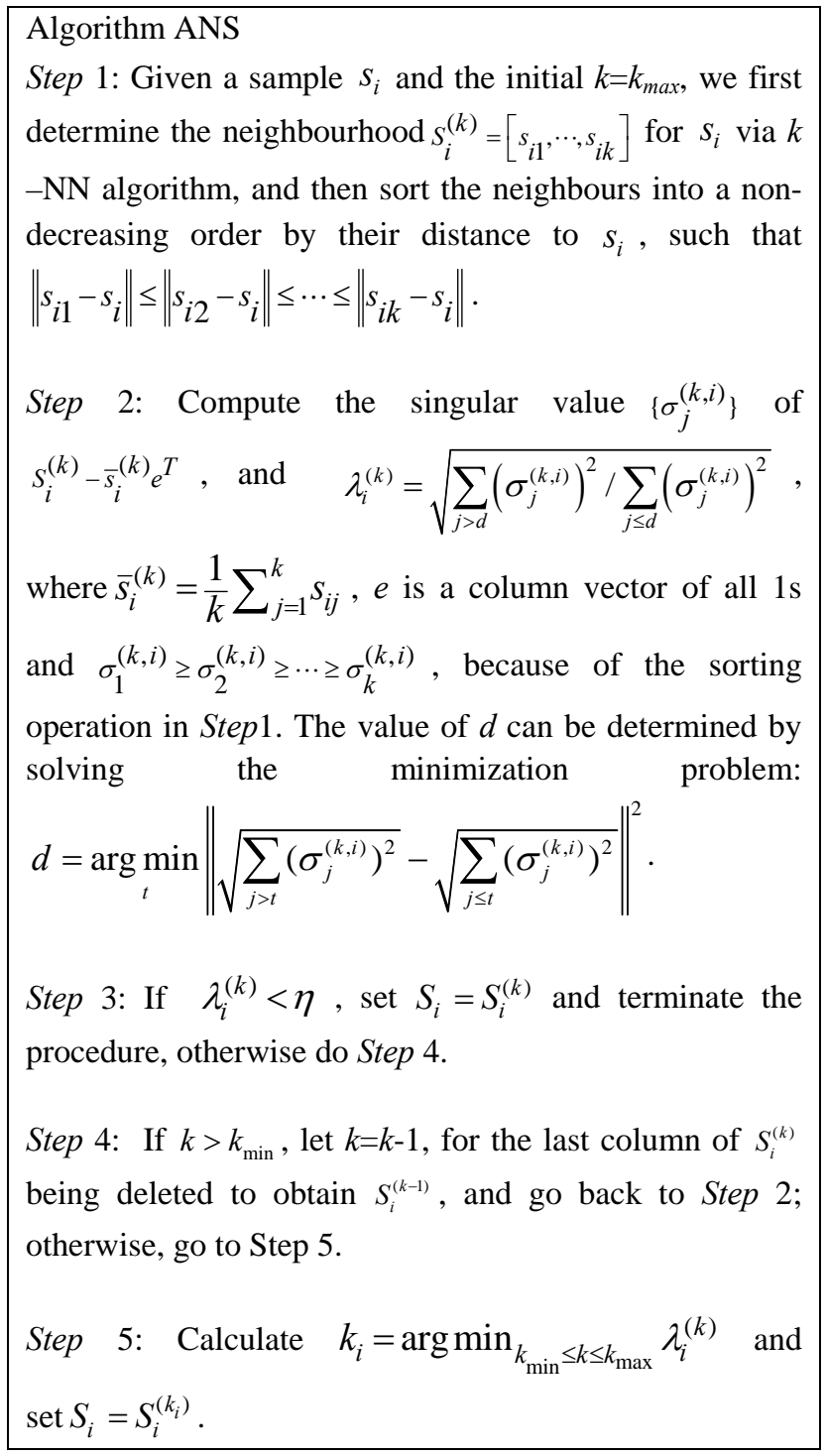

\section{FACE HALLUCINATION BASED ON ANS}

In this paper, the LR facial images are down-sampled from the corresponding HR images. We let $H=\left\{h_{i} \mid 1 \leq i \leq N\right\}$ and $L=\left\{l_{i} \mid 1 \leq i \leq N\right\}$ represent a HR face training-set and the corresponding LR face trainingset respectively, where $N$ is the number of samples. The facial images of training-set are all aligned in the preprocessing step, and then divided into overlapping image patches. Accordingly, the HR training-set and the LR one can be reformulated as $H^{p}=\left\{h_{i}^{p} \mid 1 \leq i \leq N, 1 \leq p \leq M\right\}$ and $L^{p}=\left\{l_{i}^{p} \mid 1 \leq i \leq N, 1 \leq p \leq M\right\}$ respectively, where $M$ denotes the number of patches divided in a single face image.

For an input LR facial image $l_{t}$, we first align it with the images in the LR face training-set, and then divide it into $\mathrm{M}$ overlapping patches $l_{t}=\left\{l_{t}^{1}, l_{t}^{2}, \cdots, l_{t}^{M}\right\}$. Take one patch $l_{t}^{q}(1 \leq q \leq M)$ HR reconstruction as example, the flow of the proposed face hallucination algorithm is described as follows:

Step 1: Obtain $K$ images from the LR training-set as the optimal neighbours for $l_{t}^{q}$ via the ANS algorithm. Then let $\left\{l_{t 1}^{q}, l_{t 2}^{q}, \cdots, l_{t K}^{q}\right\}$ denote the selected optimal samples which have the same patch location as $l_{t}^{q}$.

Step 2: Calculate the reconstruction weights by minimizing the reconstruction error:

$$
W_{t}^{q}=\underset{w_{t j}^{q}}{\arg \min }\left\|l_{t}^{q}-\sum_{j=1}^{K} w_{t j}^{q} l_{t j}^{q}\right\|^{2}, \text { s.t. } \sum_{j=1}^{K} w_{t j}^{q}=1
$$

where $W_{t}^{q}$ is a weight vector consisting of $M$ reconstruction weight $w_{t j}^{q}$, for $j=1,2, \ldots, M$.

Step 3: By integrating the corresponding $K$ HR samples linearly with the reconstruction weights $\left\{w_{t 1}^{q}, w_{t 2}^{q}, \cdots, w_{t K}^{q}\right\}$, the reconstructed HR patch $h_{t}^{q}$ of the LR patch $l_{t}^{q}$ can be generated. The reconstruction formulation is denoted as follows:

$$
h_{t}^{q}=\sum_{j=1}^{K} w_{t j}^{q} h_{t j}^{q}
$$

Step 4: Concatenate all the hallucinated HR patches based on the original position to produce the final HR face image $h_{t}$. Pixels of the overlapping regions in the final HR image are generated by averaging the pixel values in the overlapping regions.

\section{EXPERIMENTAL RESULTS}

The CAS-PEAL Face Database has been exploited to test our face hallucination algorithm [10], in which 1030 normal expressing images of different people under the same light condition have been selected. We aligned these face images manually and cut out the region of faces to normalize the HR facial images to $128 \times 128$ pixels. Then the corresponding LR facial images with $32 \times 32$ pixels are generated through fourfold down-sampling. In our experiments, we randomly selected 1000 images as training set. The other images are selected as test data. These parameters used in the algorithm were set as follows: $k_{\max }=60, k_{\min }=10, \eta=0.4$, the size of LR face image patch is $5 \times 5$ with 3 pixels overlapped.

To show the effectiveness of our strategy, the results of our method with those of Cubic B-Spline, [5] and [6] are compared. Considering the limited space, we just illustrate four group visual comparisons in Fig. 1 (note that the same images adopted in [5] and [6] are used here to show the effectiveness of the proposed method). 


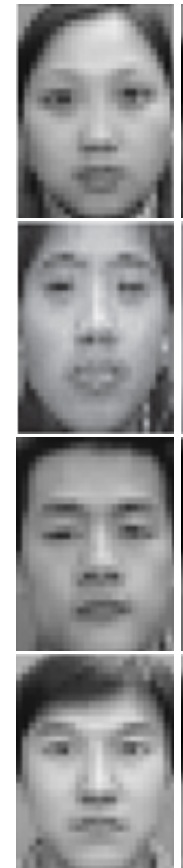

$a$
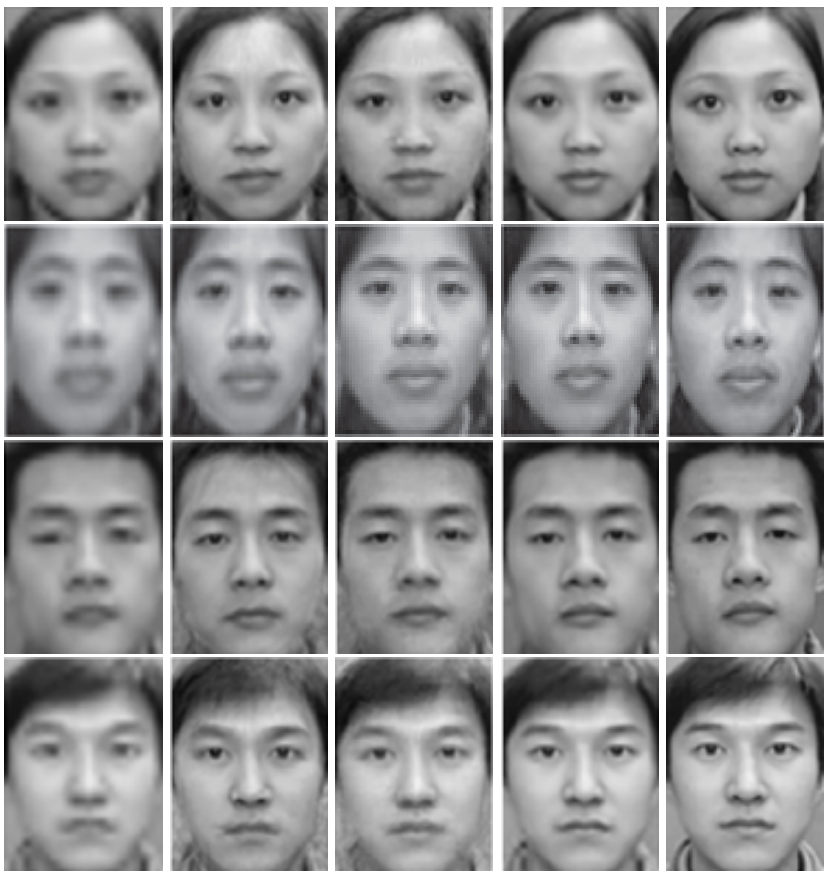

$c$

$d$

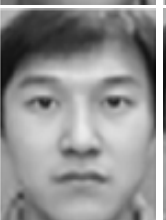

e

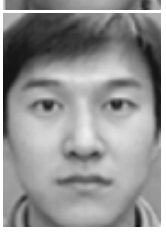

$f$

(a) Input low-resolution facial images; (b) Results of cubic B-spline interpolation; $(c)$ Results of [5];(d) Results of [6]; (e) Results of the proposed method; $(f)$ Original high-resolution face images

From Fig.1, we can see that our approach can hallucinate the HR faces with the finest details and the least artificial effects among four methods. To quantify the results, the peak signal-to-noise ratio (PSNR) values of different methods are used and provided in Fig.2. It can be obviously observed that our proposed scheme has the highest PSNR values than other methods for all face images in the test set.

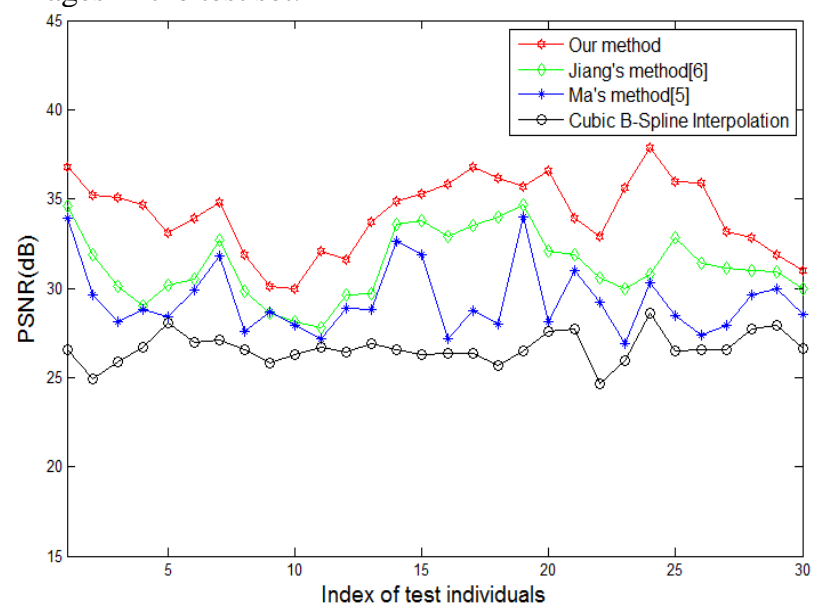

Figure 2. PSNR values of different face hallucination algorithms.

\section{V.CONCLUSION}

A novel face hallucination method based on the adaptive neighbourhood selection is proposed. By adaptively selecting the optimal neighbourhood for each face patch using the $A N S$ algorithm, it can accurately capture the local geometrical structure of the face manifold, thus improve the accuracy of the linear approximation for local geometry. To the best of our knowledge, this paper is the first to apply the $A N S$ algorithm to the manifold learning based face hallucination method. Experimental results demonstrate that the proposed algorithm outperforms the state-of-art methods in terms of visual observation and PSNR values.

\section{REFERENCES}

[1] Van, J., Image super-resolution survey, Image Vis. Comput., 24(10), pp.1039-1052, 2006.

[2] Chang, H., Yeung, D., and Xiong, Y., Super-resolution through neighbour embedding. IEEE Conf. CVPR, Washington, DC, USA, pp. 275-278, 2004.

[3] Wang, X., and Tang, X., Hallucinating face by eigentransformation,

IEEE Trans. Syst. Man Cybern. Part B, Cybern., 35(3), pp. 425-434, 2005

[4] Huang, H., He, H., Fan, X., and Zhang, J., Super-resolution of human face image using canonical correlation analysis, Pattern Recognition, 43(7), pp. 2532-2543, 2010.

[5] Xiang, M., Hiep, L., Wilfried, P., Huan, S., and Hua, C., Sparse representation and position prior based face hallucination upon classified over-complete dictionaries, Signal Processing, 92 (8), pp. 2066-2074, 2012.

[6] Jun, J., Rui, H., Zhen, H., and Tao, L., Nearest feature line embedding for face hallucination, Electronic Letters, 49(8), pp.536-538, 2013.

[7] Saul, L., Roweis, S., Think Globally, Fit Locally: Unsupervised Learning of Nonlinear Manifolds, J. Machine Learning Research, 4, pp. 119-155,2003.

[8] Zhang, Z., Zha H., Principal Manifolds and Nonlinear Dimensionality 
Reduction via Tangent Space Alignment, SIAM J. Scientific Computing, 26(1), pp. 313-338,2004.

[9] Zhen, Z., Jing, W., and Hong, Z., Adaptive Manifold Learning, IEEE Trans. Pattern Recognition and Machine Learning, 34(2), pp.253256, 2012.
[10] Gao, W., Cao, B., Shan, S., Chen, X., Zhou, D., Zhang, X., and Zhao, D., The CAS-PEAL large-scale Chinese face database and baseline evaluations, IEEE Trans. Syst. Man Cybern. Part A, Syst. Hum., 38(1), pp. 149-161, 2008. 